\title{
基于流固耦合的埋地供水管道地震响应分析
}

\author{
王丽娟，张家铭，张海潚，张 锐 \\ 河北工业大学土木与交通学院，天津 300401
}

摘 要: 为研究埋地供水管道在地震作用下的动力学特性, 以流固耦合理论为基础, 结合 ANSYS Workbench 软件, 建立埋地供水管道在地震作用下的管-水流固耦合模型, 采用四面体单元代替六面体单元 对管道内流体进行网格划分, 在施加土压力荷载和地震动力作用下, 分析管道总变形和等效应力, 探究流 体流速、工作压力、管径、埋深及壁厚等参数对管道最大等效应力响应情况. 结果表明, 在地震波加载 $4.2 \mathrm{~s}$ 时, 管道总变形和等效应力均达到最大值, 最大变形出现在管道中部, 等效应力的最大值出现在管道 的出口端; 管道的等效应力随管道的埋深、壁厚和工作压力的增加而增大, 不受流体流速的影响, 但随管 径的增加先增大后减小. 同等条件下, DN800 管道的抗震性能最佳.

关键词：市政工程；供水管道；流固耦合；动网格；地震波；有限元模拟

中图分类号: TU991 文献标志码：A doi: 10.3724/SP. J. 1249.2020.03271

\section{Seismic response analysis of buried water supply pipeline based on fluid-solid interaction}

\section{WANG Lijuan,ZHANG Jiaming, ZHANG Haixiao, and ZHANG Rui}

School of Civil and Transportation Engineering, Hebei University of Technology, Tianjin 300401, P. R. China

\begin{abstract}
In order to study the dynamic characteristics of buried water pipeline under earthquake action, we establish a fluid-solid interaction model for buried water supply pipeline under earthquake based on the theory of fluid-solid interaction and ANSYS Workbench software. The grids are generated by tetrahedral unit instead of hexahedral element. We analyze the total deformation and equivalent stress of the pipeline, and investigate the maximum equivalent stress response of the parameters such as fluid flow velocity, working pressure, pipe diameter, buried depth and wall thickness under the action of soil pressure and seismic force. The results show that the total deformation and equivalent stress of the pipeline reach the maximum when the seismic wave is loaded as $4.2 \mathrm{~s}$, and the maximum deformation occurs in the middle of the pipeline. The maximum value of the equivalent stress appears at the outlet of the pipeline; the equivalent stress of the pipeline increases with the increase of the buried depth, wall thickness and working pressure of the pipeline, and it is not affected by the fluid velocity, but increases first and then decreases with the increase of the pipe diameter. Under the same conditions, the seismic performance of DN800 pipeline is the best.
\end{abstract}

Key words : municipal engineering; water supply pipeline; fluid-solid interaction; dynamic mesh; seismic wave; finite element simulation

Citation: WANG Lijuan, ZHANG Jiaming, ZHANG Haixiao, et al. Seismic response analysis of buried water supply pipeline based on fluid-solid interaction $[\mathrm{J}]$. Journal of Shenzhen University Science and Engineering, 2020, 37(3): 271-278. (in Chinese) 
埋地供水管道是城市的生命线, 一旦在地震中 遭到破坏，将会对城市的生产生活产生巨大的影 响，并给城市的灾后重建带来不便，造成难以估量 的人员伤亡和财产损失. 现阶段中国埋地供水管道 的抗震设计存在不足, 抗震规范中管道受力模型主 要参考拟静力分析法和反应位移法 ${ }^{[1-4]}$, 这些方法 只能近似模拟地震作用下管土之间的相互作用力, 而忽略了输流管道, 特别是压力流管道与管内流体 之间的流固耦合动力作用. 管道在地震波作用下会 发生振动，管道的振动会影响到管内流体的运动， 流体流场的变化反过来又会影响到管道的动力学特 性，使得管道与管内流体之间产生流固耦合效 应 $^{[5]}$. 考虑流固耦合时, 管道的受力更接近于工程 实际状况，计算结果也更精确 ${ }^{[6]}$. 因此，将流固耦 合的理论应用于埋地供水管道抗震分析中具有一定 的理论基础和现实意义.

国内外学者针对输流管道流固耦合受力计算进 行了研究. FARHAT 等 ${ }^{[7]}$ 概述了流固相互作用问题 的三场公式，其中流体由 Euler 方程或 N-S 方程建 模，结构由有限元模型表示，流体网格是不稳定 的. 张挺等 ${ }^{[8]}$ 应用有限积分法分别配合隐式欧拉法 和拉普拉斯数值反演法，研究瞬时关阀时输流直管 轴向耦合振动响应特性. 陈坚红等 ${ }^{[9]}$ 采用 $\mathrm{C}++$ 语 言编制了充流管道单向流固耦合数值模拟程序，模 拟了管内流体压力分布以及将流体压力数据导人管 道结构中进行管道应力计算的过程. 周知进等 ${ }^{[10]}$ 利用有限元的方法对不同曲率管道的流固耦合特性 进行分析, 并研究了流固耦合作用对不同曲率管道 位置等效应力的影响. 梁军等 ${ }^{[11]}$ 通过 ADINA 软件 建立了流固耦合有限元模型, 研究了流固耦合作用 下管道的抗震性能及管内介质和流速等参数对管道 破坏的影响. 从以上研究可见, 流固耦合动力学分 析的理论研究已基本形成体系，然而并没有学者对 埋地供水管道在地震作用下的流固耦合动力学问题 进行深人的研究. 鉴于此, 本研究将流固耦合动力 学运用到埋地供水管道的地震响应分析中，以球墨 铸铁供水管道为研究对象，利用 ANSYS Workbench 有限元软件建立埋地供水管道的流固耦合模型，施 加土压力荷载和地震动力作用, 分析在管-水流固 耦合作用下管道总变形和等效应力, 并改变埋深、 管径、壁厚、流体流速及管道工作压力等参数，探 究管道等效应力的变化情况, 以期为埋地压力流管 道的抗震设计、施工方法及力学模型分析提供 参考.

\section{1 埋地供水管道流固耦合理论基础}

\section{1 流固耦合控制方程}

当考虑管水之间的流固耦合作用时，流体区域 和固体区域应各自满足其基本控制方程. 对于流体 区域，假设管内水为可轻微压缩的均匀流，考虑其 黏度，不考虑热交换过程，则其应满足流体质量守 恒平衡方程和动量守恒平衡方程 ${ }^{[12]}$.

质量守恒所对应的平衡方程为连续性方程,

$\frac{\partial \rho}{\partial t}+\frac{\partial\left(\rho u_{x}\right)}{\partial x}+\frac{\partial\left(\rho u_{y}\right)}{\partial y}+\frac{\partial\left(\rho u_{z}\right)}{\partial z}=0$

其中, $u_{x} 、 u_{y}$ 和 $u_{z}$ 分别为流体在 $x 、 y$ 和 $z$ 三个方向上 的速度分量; $t$ 为时间; $\rho$ 为密度.

动量守恒是在牛顿第二定律的基础上推导的, 动量方程在 $x$ 轴方向上的表达式 ${ }^{[13]}$ 为

$$
\begin{aligned}
\frac{\partial\left(\rho u_{x}\right)}{\partial t}+\nabla \cdot\left(\rho u_{x} \cdot V\right)= & \rho f_{x}+\frac{\partial \boldsymbol{\sigma}_{x x}}{\partial x}+\frac{\partial \boldsymbol{\tau}_{y x}}{\partial y}+ \\
& \frac{\partial \boldsymbol{\tau}_{z x}}{\partial x}-\frac{\partial \boldsymbol{P}}{\partial x}
\end{aligned}
$$

其中, $V$ 为流速矢量; $P$ 为流体压力; $f_{x}$ 为单位质量 力在 $x$ 轴方向上的分量; $\sigma_{x x}$ 为流体在 $x$ 轴方向上的 正应力; $\boldsymbol{\tau}_{y x}$ 和 $\boldsymbol{\tau}_{z x}$ 分别为沿 $y$ 和 $z$ 方向的切应力.

根据式 (2), 同理可得到动量方程在 $y$ 和 $z$ 方向 的表达式, 此处不再详述.

对于固体区域，若管内水引发管道振动或位 移, 其控制方程 ${ }^{[13]}$ 为

$$
\boldsymbol{M}_{\mathrm{s}} \cdot \frac{\mathrm{d}^{2} \boldsymbol{r}}{\mathrm{d} t^{2}}+\boldsymbol{C}_{\mathrm{s}} \cdot \frac{\mathrm{d} \boldsymbol{r}}{\mathrm{d} t}+\boldsymbol{K}_{\mathrm{s}} \cdot \boldsymbol{r}+\boldsymbol{\tau}_{s}=0
$$

其中, $M_{\mathrm{s}}$ 为固体质量矩阵; $C_{\mathrm{s}}$ 为固体阻尼矩阵; $K_{\mathrm{s}}$ 为固体刚度矩阵; $r$ 为固体位移; $\tau_{s}$ 为固体所受应 力.

将流体与固体进行耦合计算, 首先要满足流体 区域和固体区域在耦合边界上的运动学平衡方程和 动力学平衡方程 ${ }^{[14]}$. 对于埋地供水管道, 耦合面 即为管道的内壁面, 其运动学和动力学条件为

$$
\left\{\begin{array}{l}
d_{\mathrm{f}}=d_{\mathrm{s}} \\
\tau_{\mathrm{f}}=\tau_{\mathrm{s}}
\end{array}\right.
$$

其中, $d_{\mathrm{f}}$ 为流体的边界位移; $d_{\mathrm{s}}$ 为固体的边界位移; $\tau_{\mathrm{f}}$ 为流体应力; $\tau_{\mathrm{s}}$ 为固体应力.

对于无滑移壁面，流体流速 $v=d_{\mathrm{s}}$; 当耦合界 面发生相对滑移时，则有

$$
n v=n d_{\mathrm{s}}
$$

其中, $n$ 为液体流动方向. 


\section{2 流固堣合有限元求解方法}

利用有限元方法求解流固耦合问题, 需要分别 列出两者的有限元运动方程并联立. 在引人有限元 近似形函数和考虑耦合界面阻尼能量损耗的情况 下, 基于流体的连续性方程和动量方程, 得到流体 的运动方程 ${ }^{[15]}$ 为

$$
\boldsymbol{M}_{\mathrm{ef}} \cdot \ddot{\boldsymbol{P}}_{\mathrm{e}}+\boldsymbol{C}_{\mathrm{ef}} \cdot \dot{\boldsymbol{P}}_{\mathrm{e}}+\boldsymbol{K}_{\mathrm{ef}} \cdot \boldsymbol{P}_{\mathrm{e}}+\rho \boldsymbol{R}_{\mathrm{e}}^{\mathrm{T}} \cdot \ddot{\boldsymbol{U}}_{\mathrm{e}}=0
$$

其中, $P_{\mathrm{e}}$ 为流体压力; $U_{\mathrm{e}}$ 为结构位移; $M_{\mathrm{ef}}$ 为流体的 质量矩阵; $K_{\mathrm{ef}}$ 为流体的刚度矩阵; $C_{\mathrm{ef}}$ 为流体的阻尼 矩阵; $\rho R_{\mathrm{e}}$ 为耦合质量矩阵.

当固体结构受到流体施加的动水压力后, 振动 方程为

$$
M_{\mathrm{s}} \cdot \ddot{U}_{\mathrm{e}}+C_{\mathrm{s}} \cdot \dot{U}_{\mathrm{e}}+K_{\mathrm{s}} \cdot U_{\mathrm{e}}-R_{\mathrm{e}} \cdot \boldsymbol{P}_{\mathrm{e}}=\boldsymbol{F}_{e}
$$

其中, $\boldsymbol{F}_{\mathrm{e}}$ 为外部荷载矩阵; $\boldsymbol{R}_{\mathrm{e}} \cdot \boldsymbol{P}_{\mathrm{e}}$ 为流体压力荷载 矩阵.

通过联立式 (6) 与式 (7), 得到流固耦合的有 限元方程为

$$
\begin{gathered}
{\left[\begin{array}{cc}
\boldsymbol{M}_{\mathrm{s}} & 0 \\
\boldsymbol{M}_{\mathrm{fs}} & \boldsymbol{M}_{\mathrm{ef}}
\end{array}\right]\left[\begin{array}{c}
\ddot{U}_{\mathrm{e}} \\
\ddot{P}_{\mathrm{e}}
\end{array}\right]+\left[\begin{array}{cc}
C_{\mathrm{s}} & 0 \\
0 & C_{\mathrm{ef}}
\end{array}\right]\left[\begin{array}{c}
\dot{U}_{\mathrm{e}} \\
\dot{P}_{\mathrm{e}}
\end{array}\right]+} \\
{\left[\begin{array}{cc}
\boldsymbol{K}_{\mathrm{s}} & \boldsymbol{K}_{\mathrm{fs}} \\
0 & \boldsymbol{K}_{\mathrm{ef}}
\end{array}\right]\left[\begin{array}{l}
\boldsymbol{U}_{\mathrm{e}} \\
\boldsymbol{P}_{\mathrm{e}}
\end{array}\right]=\left[\begin{array}{c}
\boldsymbol{F}_{\mathrm{e}} \\
0
\end{array}\right]}
\end{gathered}
$$

式(8)表明，在流固耦合面上，各个节点具有 同样的位移和压力自由度, 当流体区域和固体区域 耦合面上的接触节点的解确定后, 通过求解式 (8) 可确定耦合面上的解向量, 从而解决流固耦合动力 学问题.

\section{2 管道流固耦合的有限元分析}

\section{1 建立管-水流固塊合模型}

运用 1.1 节中的流固耦合理论基础建立管-水流 固耦合模型. 该模型由流体相和固体相两部分组 成, 两相交界的耦合面包含管道内壁面和流体外壁 面. 建立模型时应首先应根据管道的几何尺寸建立 管道固体区域模型, 之后填充管道, 填充生成流体 区域, 从而将流固耦合面划分为两个面. 本研究根 据文献 [16], 选择研究对象为 DN500 (管道公径直 径为 $500 \mathrm{~mm}$ ) 的直管道, 长度为 $6000 \mathrm{~mm}$, 管道 外径为 $532 \mathrm{~mm}$, 壁厚取 $10 \mathrm{~mm}$.

\section{2 划分管道网格}

管道的网格划分采用六面体 8 节点单元. 管道 网格划分的精细程度会对模型后处理计算结果产生 影响, 网格划分越精细, 网格单元越密集, 计算结
果越精确, 所需计算时间也会更长. 本研究采用高 光滑度和细化跨度中心角的网格, 最小网格长 $835.66 \mathrm{~mm}$, 共划分 45796 个节点, 6776 个单元.

\section{3 流体的动网格模型}

当管道在地震波的作用下发生振动时, 流体网 格会在管道的带动下会产生收缩和膨胀. 此外, 当 固体耦合面的数据传递到流体堣合面时, 流体网格 需要作出相应的调整, 因此产生了流体的网格移动 问题. 在推导数学模型时, 可以通过将 Euler 坐标 下的 N-S 方程映射到任意拉格朗日-欧拉 (arbitrary Lagrange-Euler, ALE) 坐标系统中来解决动网格问 题, 即在流体区域中采用 Euler 单元, 对固体区域 内用 Lagrange 单元, 并在统一的 ALE 坐标系下进行 求解, 使得流体模型中的流固界面总是跟随固体的 变形而改变 ${ }^{[17]}$.

在流固耦合分析中, 流体的网格划分方式将直 接影响计算结果的收玫性和精确性. 如果依旧采用 六面体 8 节点单元的划分方式则会增加网格的畸变 率, 在计算过程中很有可能产生负体积, 导致计算 结果不收玫, 因此, 本研究采用四面体单元对流体 网格进行网格划分. 在 ANSYS Workbench 中, 通过 采用弹性光顺法和局部网格重构法来对动网格问题 进行求解, 设置弹簧屈服强度系数为 0.5 , 拉普拉 斯节点松驰系数为 0.5 , 网格重构中单元和面的最 大偏度分别为 0.4 和 0.6 , 网格尺寸重分布间隔为 10 , 共划分 3808 个节点 16902 个单元.

\section{4 设置材料参数}

模型建立且网格划分完毕后, 需要对模型的流 体区域和固体区域分别赋予对应的材料属性. 其 中, 流体材料为液态水, 密度为 $998.2 \mathrm{~kg} / \mathrm{m}^{3}$; 固 体材料为球墨铸铁, 其参数如表 $1^{[16]}$.

表 1 球墨铸铁管材料参数 ${ }^{[16]}$

Table 1 Material parameters of ductile cast iron pipeline

\begin{tabular}{cccccc}
\hline 材料 & $\begin{array}{c}\text { 密度/ } \\
\left(\mathrm{kg} \cdot \mathrm{m}^{-3}\right)\end{array}$ & $\begin{array}{c}\text { 杨氏模 } \\
\text { 量/GPa }\end{array}$ & 柏松比 & $\begin{array}{c}\text { 屈服强 } \\
\text { 度/MPa }\end{array}$ & $\begin{array}{c}\text { 抗拉强 } \\
\text { 度/MPa }\end{array}$ \\
\hline $\begin{array}{l}\text { 球墨 } \\
\text { 铸铁 }\end{array}$ & 7300 & 150 & 0.30 & 300 & 420 \\
\hline
\end{tabular}

在 ANSYS Workbench 中需要在 Engineering Data 模块下设置与定义球墨铸铁材料, 并输人材料参 数, 以便于在建立模型后将材料属性赋予给管道固 体区域.

\section{5 模型的边界条件}

图 1 为埋地供水管道的受力示意图. 其中, $M$ 为管道覆土厚度; $D^{*}$ 为管顶至管道开挖沟槽底面的 
距离. 本研究模型中，设置管道的两个端面为固定 约束, 数值模拟计算范围等同于管道的计算长度.

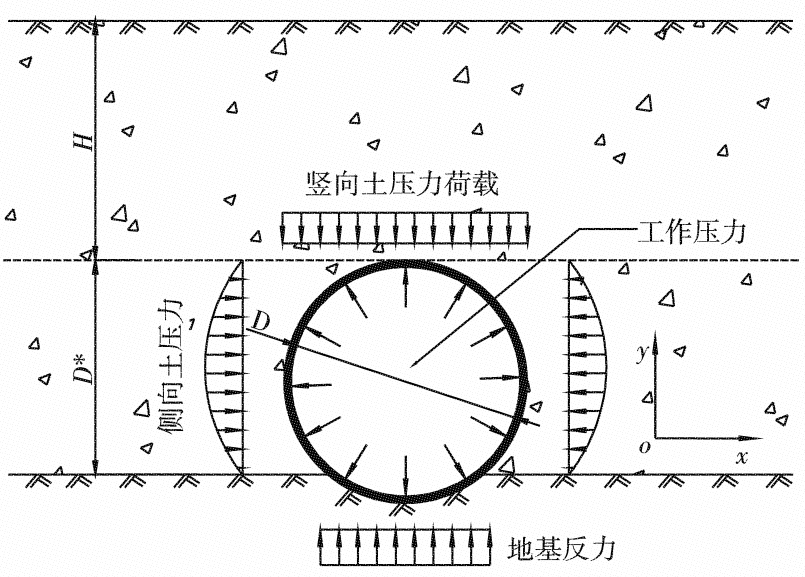

图 1 埋地供水管道受力示意图

Fig. 1 Stress diagram of buried water supply pipeline

取管道埋深为 $2 \mathrm{~m}$ ，并考虑管道与管内水自重 的影响. 在静载作用下，管道左右两侧的土压力相 互平衡，地基反力的大小与坚向土压力荷载、管道 与管内流体自重及地基承载力的大小有关.

为简化计算，本研究将作用在管道上的土压力 荷载等效为均布荷载, 并忽略侧向土压力和地基反 力，即只考虑坚向土压力荷载对管道的影响. 坚向 土压力荷载作用在管道外表面的上半部分，计算 式 $^{[18]}$ 为

$$
F_{\mathrm{sv}, \mathrm{k}}=C_{\mathrm{d}} \gamma_{\mathrm{s}} H_{\mathrm{s}} B_{\mathrm{c}}
$$

其中, $F_{\mathrm{sv}, \mathrm{k}}$ 为等效坚向土压力荷载; $C_{\mathrm{d}}$ 为土压力系 数; $\gamma_{\mathrm{s}}$ 为回填土重力密度; $H_{\mathrm{s}}$ 为管顶至地面的覆土 高度; $B_{\mathrm{c}}$ 为管道的外径.

对于管内流体部分，设置管内流体为标准 $k-\varepsilon$ 的湍流模型, 忽略流体在运动过程中与管壁产生的 热量交换，并分别定义流速人口端面和流速出口端 面，与管道端面平齐，且为自由平面，不设置约 束. 在出人口端面上, 流体速度为绝对速度, 大小 为 $1.2 \mathrm{~m} / \mathrm{s}$, 方向为垂直于边界, 并取湍流强度为 $5 \%$ ，湍流黏度比为 10 .

当供水管道处于工作状态时，管内为压力流， 取工作压力为 $0.6 \mathrm{MPa}$. 对于流固耦合面, 设置流 体在流经管道壁面时不与管道壁面产生滑移，并取 耦合面的粗糙度常数为 0.5 .

\section{6 施加地震动力作用}

本研究选取国内外结构抗震设计常用的美国 Northridge 地震中观测到的地震波数据, 并取前 $10 \mathrm{~s}$ 计算时间，作为埋地供水管道模型的地震动力荷
载 ${ }^{[19]}$, 加速度时程曲线如图 2.

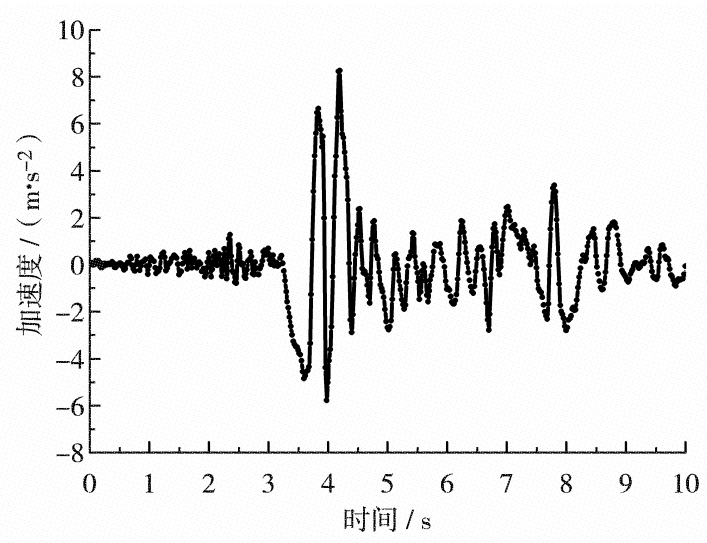

图 2 地震波的加速度-时间曲线

Fig. 2 Acceleration-time curve of seismic wave

该地震的震级为里氏 6.7 级, 地震波峰值加速 度的平均值在 $0.3 \mathrm{~g}$ 以上, 前 $10 \mathrm{~s}$ 的最大地震加速 度约为 $0.8 \mathrm{~g}$, 按照中国抗震设计规范的标准, 对该 地震波作用下的构筑物进行抗震设计时，需要按照 抗震设防烈度为 8 度来考虑 ${ }^{[20]}$.

在 ANSYS Workbench 中，需要利用 Transient Structural 模块将地震动力作用施加在管道上. 设置 总作用时间为 $10 \mathrm{~s}$, 以 $0.02 \mathrm{~s}$ 为 1 个时间步长，输 人每个时间点所对应的地震波加速度，共输人 500 组数据，对应加速度时程曲线上的每个点，便可以 模拟供水管道在地震波作用下的振动情况. 地震波 的传播方向设置为沿着管轴方向和垂直于管轴方 向，同时进行传播.

\section{7 流固耦合模型求解}

管道和管内流体之间的耦合是一种双向流固耦 合，计算原理可参考式(8). 采用牛顿-拉夫森迭代 法，在每个时间步上将固体区域的计算结果加载至 流体，引起流体流场变化后重新计算流体，将新的 结果重新作用在固体区域上，以此反复迭代，得到 最终的收玫结果. 在 ANSYS Workbench 中, 需要将 设置好的流体区域和固体区域通过 System Coupling 模块进行迭代计算，并设置计算时间步长、流体与 固体的迭代顺序以及总计算时长，从而实现模型的 求解. 迭代求解法计算量大、耗时较长，对计算机 的性能要求较高，在模拟过程中需要考虑迭代收敛 性和计算效率问题.

\section{3 流固耦合模拟结果分析}

当地震波加载至 $4.2 \mathrm{~s}$ 时，管道的总变形达最 
大值, 云图如图 3. 由图 3 可见, 最大值出现的位 置在管道中部, 这是因为管道两端固接, 管道中部 的位移约束小, 在地震波作用下振幅较大, 所以相 较于管道其他位置, 管道中部的总变形最大.

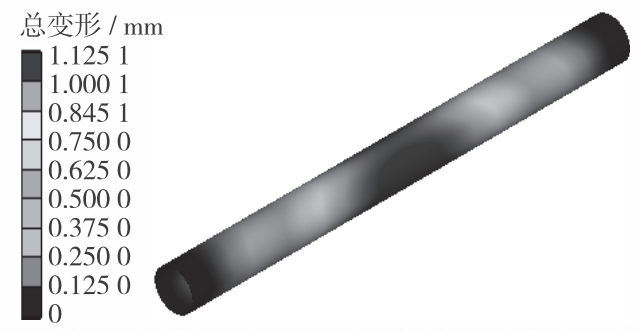

图 $3 t=4.2 \mathrm{~s}$ 时管道的总变形云图

Fig. 3 (Color online) Total deformation nephogram of pipeline at $4.2 \mathrm{~s}$

图 4 为埋地供水管道模型在地震作用下管段中 部的总变形随时间的变化曲线. 从总体上看，管道 中部下端的变形比上端的变形高 $3.8 \%$, 在地震刚 开始的一段时间内, 由于地震波的加速度数值较 小, 变化幅度不大, 所以管道产生的振动也不明 显, 管道产生的变形主要来自于管和水的自重以及 管顶的坚向土压力荷载. 随着地震的持续进行, 地 震加速度逐渐达到峰值, 管道的变形受地震作用的 影响较为显著, 且变化趋势与地震波的加速度时程 曲线变化基本一致.

同样当地震波加载至 $4.2 \mathrm{~s}$ 时, 管道的等效应 力也达到最大值, 云图如图 5. 最大等效应力的位 置在管道的出口, 这是由于模型假定管道两个端面 不发生变形和移动, 当管道中部产生变形后, 距离 中部越远的管段所产生的应力就会越大, 并且地震 波作用方向为管道出口, 因此管道出口的等效应力 要比管道人口更大.

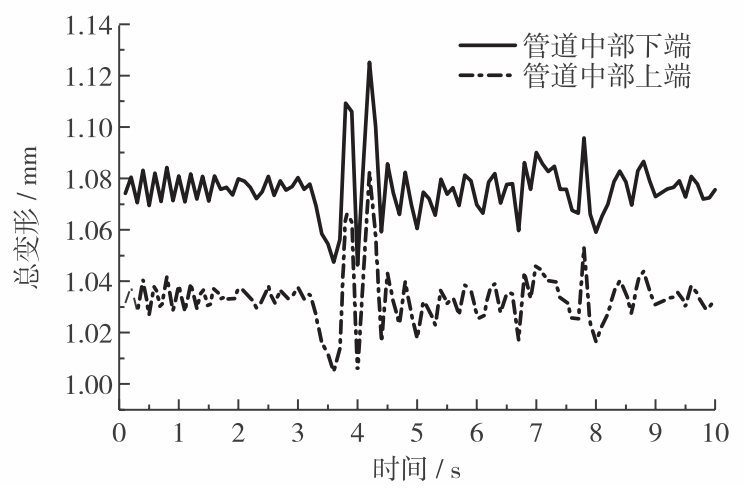

图 4 管道中部总变形时程曲线

Fig. 4 (Color online) Time history curve of total deformation in the middle of the pipeline

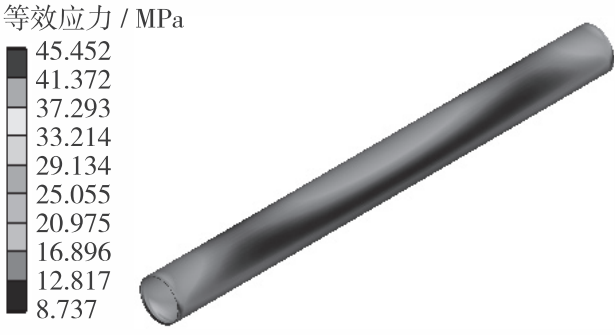

图 $5 t=4.2 \mathrm{~s}$ 时管道的等效应力云图

Fig. 5 (Color online) Equivalent stress nephogram of pipeline at $4.2 \mathrm{~s}$

在模型的计算过程中, 由于管道一直处在振动 状态，管-水耦合界面也相应发生扩张和收缩. 流 体的动网格算法根据流体边界的变化不断地更新网 格, 通过拉伸或重新生成网格来适应流体区域的变 化, 确保管-水耦合界面处于高度耦合状态, 并且 能够有效地在两相之间传递计算数据.

\section{4 影响管道等效应力变化的因素}

\section{1 管内流体流速的影响}

其他条件不变, 改变管道内流体的流速分别为 $0.5 、 1.5 、 2.5 、 3.5$ 和 $4.5 \mathrm{~m} / \mathrm{s}$, 探究流速变化对 地震作用下埋地管道等效应力变化的影响, 结果如

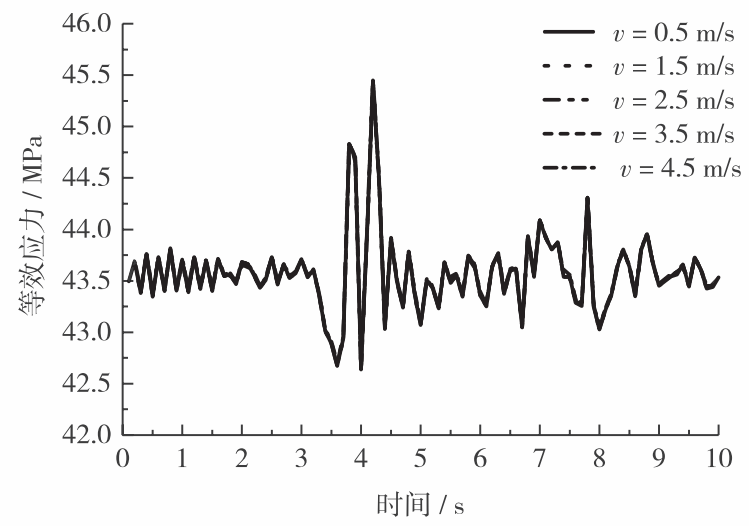

图 6 不同流速下管道的等效应力

Fig. 6 (Color online) Equivalent stress of pipeline under different flow velocities

图 6. 从图 6 可以看出, 管道的等效应力并没有因 为流速的改变而发生明显的变化, 这是因为管道内 流体的密度和体积不随流速的改变而改变, 在忽略 流体惯性力的条件下, 流体质量不变, 对管道产生 的作用力也不变, 所以管道等效应力也不变, 这与 文献[11]中的结论相符. 因此, 可以认定在合理的 
流速范围内, 管道内流体流速变化并不影响地震作 用下管道的等效应力大小.

\section{2 管道工作压力的影响}

其他条件不变，分别选取管道工作压力为 0.4 、 $0.6 、 0.8 、 1.0$ 和 $1.2 \mathrm{MPa}$ ，对比管道在不同工作 压力下的等效应力, 结果如图 7. 由图 7 可见, 随 着工作压力的增大, 管道的等效应力也随之增加. 这是因为工作压力增大时, 管道内壁会受到动水压 力的作用发生变形, 管道环向应力增大, 致使管道 等效应力增大. 但是, 管道工作压力从 $0.4 \mathrm{MPa}$ 至 $1.2 \mathrm{MPa}$ 增长了 3 倍, 等效应力只增加了 $31.4 \%$. 对于采用柔性连接的 DN500 球墨铸铁管，允许工作 压力约为 $4.4 \mathrm{MPa}$, 因此在安全工作压力下, 管道 工作压力增大会使管道在地震作用下的等效应力增 大，但等效应力增长比率远小于工作压力增长的 幅度.

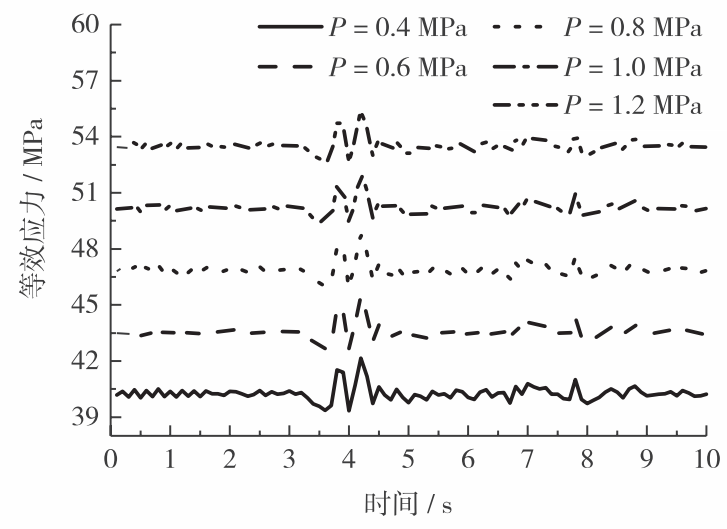

图 7 不同工作压力下管道的等效应力

Fig. 7 (Color online) Equivalent stress of pipeline under different working pressure

\section{3 管道埋深与管径的影响}

图 8 给出了在壁厚均为 $10 \mathrm{~mm}$, 埋深分别为 2 、 $3 、 4 、 5$ 和 $6 \mathrm{~m}$ 时, DN300、DN500、DN800、 DN1000 和 DN1200 五种不同规格管道在地震波加 载过程中最大等效应力的变化情况. 从图 8 可见, 不论对于何种公称直径的管道, 最大等效应力均随 管道埋深的增加而增大. 这是因为在地震发生后, 地表裂缝呈楔形, 土体位移随埋深增大而减小 ${ }^{[21]}$, 在不考虑地面面波和场地大变形的前提下，当埋深 增大时，作用在管道上方的坚向土压力荷载增大， 在地震的作用下土压力的增大会对管道振动的约束 作用增大，导致管道等效应力增大.

此外, 随着管道公称直径的增大, 管道在地震 中所受到的最大等效应力先减小后增大，其中，

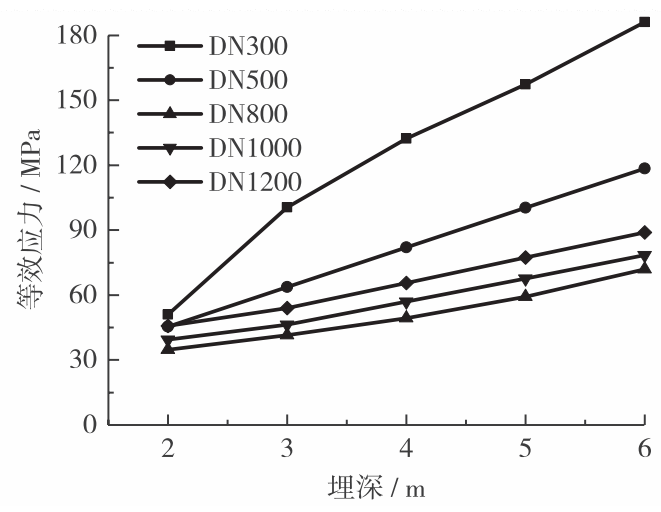

图 8 不同埋深和不同公称直径下管道的最大等效应力

Fig. 8 (Color online) Maximum equivalent stress of pipeline with different buried depth and different nominal diameter

DN800 的管道在其他条件相同的情况下等效应力最 小. 这说明了管径的增大有利于提高管道的抗震性 能, 但是随着管径的增大, 管道和管水的重力也增 大, 自重对等效应力的影响逐渐占据主导地位, 在 地震过程中振动惯性力加大, 故当管道公称直径超 过 $800 \mathrm{~mm}$ 时，管道等效应力反而增大.

\section{4 管道壁厚的影响}

按照球墨铸铁管的规格标准，不同公称直径的 管道所采用的管道壁厚不同. 甚至在相同公称直径 的管道下，管道壁厚也有着不同的取值. 表 2 给出 了不同公称直径球墨铸铁管在工程上常用的壁厚取 值 ${ }^{[16]}$. 其中, $\mathrm{K} 8 、 \mathrm{~K} 9$ 和 $\mathrm{K} 10$ 为球墨铸铁管的壁厚 等级.

表 2 不同公称直径下管道的壁厚 ${ }^{[16]}$

Table 2 Wall thickness of pipeline with different nominal diameters ${ }^{[16]}$ $\mathrm{mm}$

\begin{tabular}{cccc}
\hline \multirow{2}{*}{ 公称直径 } & \multicolumn{3}{c}{ 壁厚 } \\
\cline { 2 - 4 } & $\mathrm{K} 8$ & $\mathrm{~K} 9$ & $\mathrm{~K} 10$ \\
\hline 300 & 6.4 & 7.2 & 8.0 \\
500 & 8.0 & 9.0 & 10.0 \\
800 & 10.4 & 11.7 & 13.0 \\
1000 & 12.0 & 13.5 & 15.0 \\
1200 & 13.6 & 15.3 & 17.0 \\
\hline
\end{tabular}

为更直观地反映管道壁厚对管道等效应力的影 响，保持其他条件不变，在不同公称直径下管道分 别取标准壁厚和统一壁厚，标准壁厚取表 2 中的 $\mathrm{K} 8$ 列数据, 统一壁厚取 $10 \mathrm{~mm}$, 并将计算结果作 对比.

图 9 显示了不同管径下管道的等效应力. 由图 
9 可见，在同一公称直径的管道中，等效应力越小， 所对应管道壁越厚，且壁厚差距越大，等效应力差 越大．这是因为管壁越厚，管道的刚性越大，管道 在地震波影响下的振动强度越小，管内流体对管壁 的冲击力减弱，管道的流固耦合作用力降低，管道 等效应力减小, 所以厚壁埋地供水管道的抗震性能 比薄壁埋地供水管道更好. 当取统一壁厚时, 可明 显看出 DN800 的管道等效应力最小，印证了 4.3 节 的结论. 因此,在满足规范设计要求以及经济合理的 范围内，在管道的抗震设计中应尽量采用管道公称 直径在 $800 \mathrm{~mm}$ 左右且管壁较厚的管道.

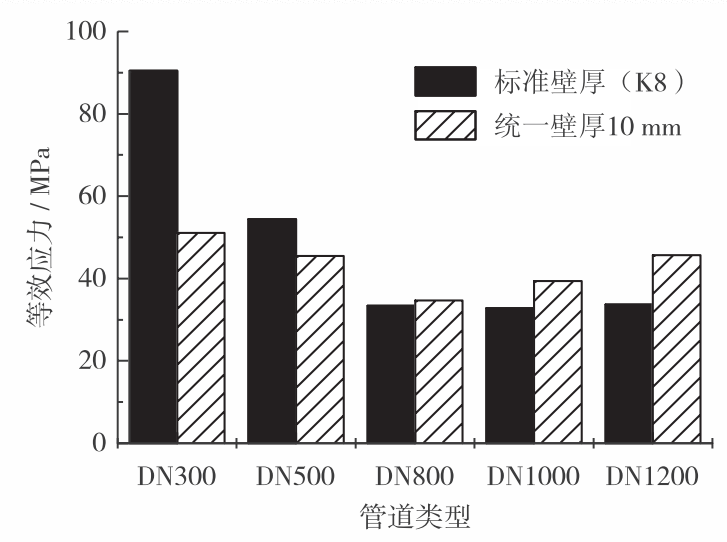

图 9 不同管径下管道的等效应力

Fig. 9 (Color online) Equivalent stress of pipeline with different diameters

\section{5 结 论}

基于流固耦合理论，建立了地震作用下埋地供 水管道管-水流固耦合模型，实现了管道在地震动 力作用下的流固耦合计算及抗震模拟. 改变管内水 的流速、管道工作压力、管道埋深、管径以及壁 厚，对比了不同参数影响下管道最大等效应力的变 化情况，可知:

1）采用流固耦合模型进行管道地震响应分析, 与传统分析方法相比省略了很多假设性条件, 具有 模型简易，计算过程简便等优势，得到的结果与现 有研究相符, 佐证了本研究模型在管道抗震方面的 可行性和正确性, 是流固耦合仿真研究的一种新运 用途径，可为现阶段埋地供水管道抗震设计规范的 补充，也可以推广至输油、输气等压力流管道的抗 震计算中.

2）埋地供水管道在地震波的作用下会发生受 迫振动, 当地震波的加速度达到峰值时, 管道的变 形和等效应力也达到了峰值, 最大变形出现在管道
模型的中部，最大等效应力出现在管道的出口端.

3）在地震波作用下，管道内流体的流速对管 道等效应力几乎没有影响，管道工作压力的增大会 使得管道等效应力增大，但影响不明显. 所以在一 般情况下，可以认为管内流体流速对管道抗震性能 没有影响，工作压力变化对管道的抗震性能有较小 的影响.

4）管道深埋以及增大管道壁厚，都可以减小 管道受震下的等效应力. 除此之外，管径变化对管 道等效应力有较大影响. 在管径较小时，管道的等 效应力很大，随着管径的增大，管道的等效应力减 小. 采用 DN800 管道时管道等效应力最小，管径继 续增大，管道等效应力随之增大，因此在管道设计 上需要考虑最佳的管径取值.

\section{基金项目：河北省博士后基金资助项目（B2013005003） \\ 作者简介: 王丽娟 $(1972-)$, 河北工业大学高级工程师、博士. 研究方向：管道振动特性与市政管网抗震分析. E-mail： wlj@ hebut.edu.cn 张家铭 (1995-), 河北工业大学硕士研究生. 研究方 向：管道抗震. E-mail: 719042137@ qq.com 王丽娟、张家铭为共同第一作者. \\ 引 文: 王丽娟, 张家铭, 张海潇, 等. 基于流固耦合的埋地供 水管道地震响应分析 $[\mathrm{J}]$. 深圳大学学报理工版， 2020 , $37(3)$ : 271-278.}

\section{参考文献 / References :}

[ 1 ] CHENG K M, WANG R L. Seismic response behavior of buried pipelines [ J ]. Journal of Pressure Vessel Technology, 1979, 101 (1) : 21-30.

[ 2 ] HETENYI M. Beams on elastic foundationtheory with applications in the fields of civil and mechanical engineering [ M ]. Michigan: University of Michigan Press, 1958.

[ 3 ] 范文亮, 姚娟娟, 张春涛, 等. 埋地管道的横向静力分 析耦合模型及其解耦条件 $[\mathrm{J}]$. 计算力学学报, 2013 , 30 (3) : 437-443.

FAN Wenliang, YAO Juanjuan, ZHANG Chuntao, et al. A coupling model and uncoupling condition of ring static analysis for buried pipes $[\mathrm{J}]$. Chinese Journal of Computational Mechanics, 2013, 30 (3) : 437-443. (in Chinese)

[ 4 ] 黄强兵, 彭建兵. 基于地震波人射角的地下管道地震 应力计算 $[\mathrm{J}]$ 。地下空间与工程学报，2008，4(5)： 979-984.

HUANG Qiangbing, PENG Jianbing. Calculation about earthquake stress for underground pipeline based on the incident angle of earthquake wave $[\mathrm{J}]$. Chinese Journal of Underground Space and Engineering, 2008, 4 (5) : 979- 
984. (in Chinese)

[ 5 ] ZHANG J, CHILDRESS S, LIBCHABER A, et al. Flexible filaments in a flowing soap film as a model for onedimensional flags in a two-dimensional wind $[\mathrm{J}]$. Nature, 2001, 408(6814) : 835-839.

[ 6 ] WYLIE E B, STREETER V L, WIGGERT D C. Fluid transients $[\mathrm{J}]$. Journal of Fluids Engineering, 1980, 102 (3) : 384-385.

[ 7 ] FARHAT C, GEUZAINE P, BROWN G. Application of a three-field nonlinear fluid-structure formulation to the prediction of the aeroelastic parameters of an F-16 fighter [J]. Computers \& Fluids, 2003, 32 (1) : 3-29.

[ 8 ] 张 挺, 郭晓梅, 谭志新, 等. 基于有限积分法的输流 直管轴向流固耦合有限积分法数值模拟 $[\mathrm{J}]$. 振动工 程学报, 2019, 32(1) : 160-167.

ZHANG Ting, GUO Xiaomei, TAN Zhixin, et al. Numerical simulation on FSI characteristic of fluidconveying straight pipe by finite integration method $[\mathrm{J}]$. Journal of Vibration Engineering, 2019, 32 (1) : 160167. (in Chinese)

[9]陈坚红, 周天情, 盛德仁, 等. 充流管道单向流固耦合 数值模拟自动化研究 $[\mathrm{J}]$. 动力工程学报, 2012, 32 (8) : 612-616.

CHEN Jianhong, ZHOU Tianqing, SHENG Deren, et al. Automation of numerical simulation on one-way fluidstructure interaction of fluid-filled pipeline $[\mathrm{J}]$. Journal of Power Engineering, 2012, 32(8) : 612-616. (in Chinese)

[10］周知进, 何 星, 戴哲冰. 不同曲率情况下的液压管道 流固耦合特性仿真研究 $[\mathrm{J}]$. 振动与冲击, 2017, 36 (5) : 214-220.

ZHOU Zhijin, HE Xing, DAI Zhebing. Simulation for fluid-solid interaction characteristics of hydraulic pipes with different curvatures $[\mathrm{J}]$. Journal of Vibration and Shock, 2017, 36(5) : 214-220. (in Chinese)

[11] 梁 军, 朱庆杰. 流固耦合作用下流体对管道抗震性 能的影响分析 $[\mathrm{J}]$ 。 世界地震工程, 2007, 23(3) : 2328 .

LIANG Jun, ZHU Qingjie. Influence of liquid on earthquake resistance of pipeline under fluid-solid interaction [J]. World Earthquake Engineering, 2007, 23(3) : 23 28. ( in Chinese)

[12] 欧益宏, 李 润, 袁广强, 等. 基于流固耦合的立式拱 顶储罐油气爆炸数值模拟 $[\mathrm{J}]$. 振动与冲击, 2018, 37 (22) : 236-242.

OU Yihong, LI Run, YUAN Guangqiang, et al. Numerical simulation on the gasoline-air mixture explosion in vertical dome roof oil tanks with consideration of the fluid-structure coupling effect $[\mathrm{J}]$. Journal of Vibration and Shock, 2018, 37(22) : 236-242. (in Chinese)

[13] VERSTEEG H K, MALALASEKERA W. An introduction to computational fluid dynamics: the finite volume method [M]. New York: Wiley, 1995.

[14] 魏 纲, 徐日庆, 黄 斌. 长距离顶管管道的失稳分析 [J]. 岩石力学与工程学报, 2005, 24(8) : 1427-1432. WEI Gang, XU Riqing, HUANG Bin. Analysis of instability of long distance pipe jacking pipe $[\mathrm{J}]$. Chinese Journal of Rock Mechanics and Engineering, 2005, 24 (8) : 1427-1432. (in Chinese)

[15] CRAGGS A. A finite element model for acoustically lined small rooms $[\mathrm{J}]$. Journal of Sound and Vibration, 1986, $108(2)$ : 327-337.

[16] 沈世杰, 王大龄, 王长祥. 给水排水工程结构设计手册 [M]. 中国建筑工业出版社, 2007.

SHEN Shijie, WANG Daling, WANG Changxiang. Structural design manual for water supply and drainage engineering $[\mathrm{M}]$. China Building Industry Press, 2007. (in Chinese)

[17] 张 雄, 陆明万, 王建军. 任意拉格朗日-欧拉描述法 研究进展 $[\mathrm{J}]$. 计算力学学报, 1997, 14(1) : 91-102.

ZHANG Xiong, LU Mingwan, WANG Jianjun. Advances in the study of arbitrary lagrangian-euler description $[\mathrm{J}]$. Chinese Journal of Computational Mechanics, 1997, 14 (1) : 91-102. (in Chinese)

[18］GB 50332-2002 给水排水工程管道结构设计规范 $[\mathrm{S}]$.

GB 50332-2002 Structural design code for pipelines water supply and waste water engineering $[\mathrm{S}]$. (in Chinese)

[19］朱文正. 多跨简支梁桥加固措施的抗震性能评估 $[\mathrm{J}]$. 深圳大学学报理工版, 2015, 32(2): 152-161.

ZHU Wenzheng. Seismic performance evaluation of retrofit measures for multi-span simple-supported bridges $[\mathrm{J}]$. Journal of Shenzhen University Science and Engineering, 2015, 32(2) : 152-161. (in Chinese)

[20] 安 韶, 王 威, 马东辉. 城市供水管道性态抗震设计 方法 $[\mathrm{J}]$. 北京工业大学学报, 2019, 45(5) : 36-43.

AN Shao, WANG Wei, MA Donghui, et al. Performancebased seismic design method of urban water supply pipe network $[\mathrm{J}]$. Jornal of Beijing University of Technology, 2019, 45(5) : 36-43. (in Chinese)

[21] 陈 勇, 杨庆华. 埋深对地下管道抗震设计的影响 [J]。世界地震工程, 2015, 31 (2) : 275-280。

CHEN Yong, YANG Qinghua. Depth on seismic design of buried pipelines $[\mathrm{J}]$. World Earthquake Engineering, $2015,31(2): 275-280$. (in Chinese)

【中文责编：坪 梓; 英文责编：之 聿】 